\title{
The Channel Compressive Sensing Estimation for Power Line Based on OMP Algorithm
}

\author{
Yiying Zhang, ${ }^{1}$ Kun Liang, ${ }^{1}$ Yeshen $\mathrm{He}^{2}{ }^{2}$ Yannian $\mathrm{Wu},{ }^{2} \mathrm{Xin} \mathrm{Hu}^{2}$ and Lili Sun \\ ${ }^{1}$ College of Computer Science and Information Engineering, Tianjin University of Science \& Technology, Tianjin, China \\ ${ }^{2}$ China Gridcom Co., Ltd, Shenzhen, Guangdong, China \\ Correspondence should be addressed to Kun Liang; liangkun@tust.edu.cn
}

Received 12 November 2016; Accepted 28 March 2017; Published 23 April 2017

Academic Editor: Hui Cheng

Copyright (c) 2017 Yiying Zhang et al. This is an open access article distributed under the Creative Commons Attribution License, which permits unrestricted use, distribution, and reproduction in any medium, provided the original work is properly cited.

\begin{abstract}
Power line communication (PLC) can collect information by power line which increases the coverage and connectivity of the smart grid. In this paper, we analyze the transmission characteristics of the power line channel and model it with mathematics channel. The multipath effect of the power line channel is studied with a novel technology named compressive sensing herein. We also proposed a new method to the power line channel estimation based on compressive sensing. We can collect and extract the effective parameters of the power line channel to storage, which only take very little storage space. The simulation results show that the proposed approach can reduce the amount of processing data in the digital signal processing module and decrease the requirement for the hardware.
\end{abstract}

\section{Introduction}

Industry 4.0 employs CPS (Cyber-Physical System) to promote industrial production with interconnection and intelligence. And various communication technologies provide important support for the CPS. Power Line Communication, as a special communication technology, not only is designed to transmit electrical energy, but also is used to transmit multiple types of information [1]. However, due to the fact that the power line network is designed for the transmission of electrical energy and works in high electromagnetic phenomena environment, the characteristics of power line channel are different from other conventional communication channel greatly. Power line noise is very complex. It is not a single Gaussian white noise in the other usual communication environments and includes colored background noise and periodic impulse noise (asynchronous or synchronized), as shown in Figure 1.

According to the random in or out access of electrical equipment, PLC has a strong time-varying characteristic [2, 3]. The channel state information is essential for the relevant data detection, quantification, and interference suppression. Therefore, we need to further analyze and conduct research on the transmission characteristics and estimation methods of power line channel [4-6]. Generally, the traditional channel estimation method has three ways. Nonblind channel estimation is the most traditional channel estimation method, which mainly makes use of the pilot signal response channel's features on transmitting terminal. Blind channel estimation is a kind of channel estimation method which does not need to send pilot signal, Semiblind channel estimation is a compromise method between the above two methods [7]. The traditional channel estimation method requires a high speed analog to digital converter. In order to accurately estimate the channel characteristics on receiving terminal, we need to send long pilot signal and collect large sample data. There is no doubt that the hardware complexity and cost of the receiving terminal will increase.

Different from other areas of data compression for universal video, voice, image number, and so on, in order to be able to reliably analyze the power grid state, the data of power system stored after compression must be able to retain the perturbed feature quantities for each frequency band of the power quality [8]. In [8-10], authors adopt the wavelet transform to apply the power quality disturbance data compression and obtained a certain degree of compression. However, 


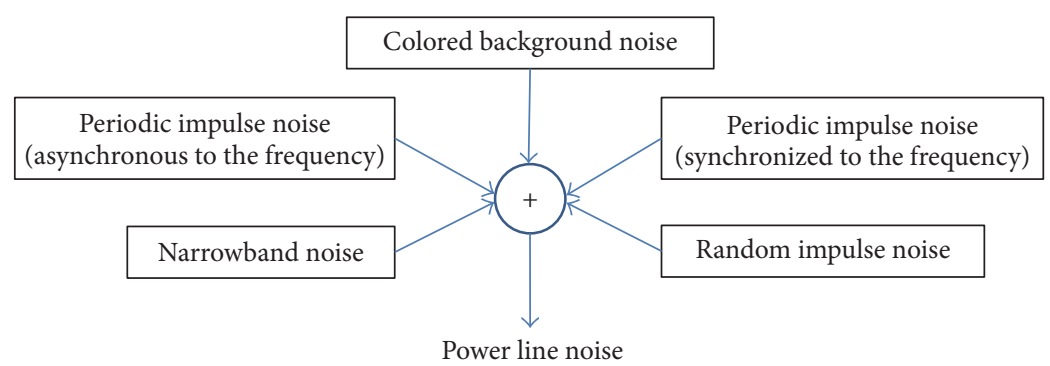

Figure 1: Power line noise.

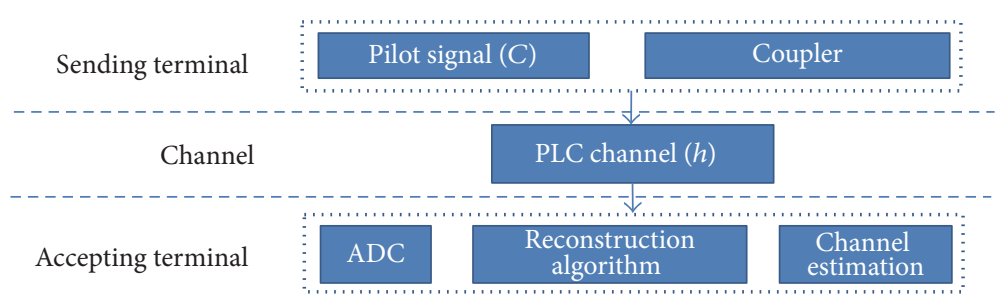

FIGURE 2: Block diagram of PLC estimation model.

the wavelet transform algorithm is complex, computationally intensive, real-time, and difficult to apply in real-time power quality monitoring system; meanwhile, the wavelet function is not unique, often using different wavelet analysis of the same signal analysis results which may vary greatly without adaptability.

Compression sensing includes two parts: the signal is measured on the measurement vector, and the signal is reconstructed by the measured value $[11,12]$. Compression sensing theory shows that if the original signal is sparse on a certain base, the sampling frequency can be greatly reduced, and the original signal can be reconstructed exactly when the constrained equidistant condition between the observation matrix and the transform base is satisfied. The signal is sampled and compressed.

Therefore, researcher suggests that transmission characteristics of the power line channel are time-varying linear channel. We can estimate it based on OFDM pilot signal. However, this channel estimation mechanism ignores ADC device requirements on the receiving terminal. In the wireless communication scenario, a method of wireless channel estimation is based on compressive sensing. By orthogonal matching pursuit (OMP) algorithm on receiving terminal, we reconstruct the transmission characteristics of wireless channel [13-15]. This paper proposed an approach for the power line channel estimation based on compressive sensing. We analyzed the sparse characteristic of power line channel. Send the appropriate pilot signal by compressive sensing technology from the transmitting terminal. After the power line channel delay and attenuation, we extracted the effective features of power line channel to finish the power line channel estimation.

The rest of this paper is organized as follows. Section 2 presents PLC channel estimation model. Section 3 shows compressive sensing estimation channel characteristics. Section 4 describes the simulation in detail and Section 5 evaluates our solution and gives the conclusion.

\section{Power Line Communication Channel Estimation Model}

Power line communication channel estimation model includes two parts: transmitting terminal and receiving terminals. Transmitting terminal is used to send appropriate pilot signal, amplified and coupled to the power line through the coupling circuit and then is influenced by the actual power line channel environment. Channel estimation at the receiver can get the transmission characteristics of power line channel.

As shown in Figure 2, the transmission signal includes the effective signal and pilot signal, amplified and coupled to the power line through the coupling circuit. The signal is attenuated by the power line channel and the interference of the noise. Through a coupling circuit, receiving terminal does the electrical isolation and receiving and then starts the digital signal processing after A/D (analog-to-digital signal conversion) by ADC [16].

Usually, those residential areas are generally used in the combination of radial and trunk distribution mode. There are a large number of nodes in the power line network, such as the branch structure and the impedance mismatch [17], which are shown in Figure 3.

These nodes cause the transmission signal on the power line to not be able to reach the receiving node directly from the sending node. There will be reflected and standing waves on different paths. The final receiving device received the superimposed signal via reflected and standing wave in the different paths. This makes the power line channel cause multipath effect, and the transmission characteristics show a certain frequency selective fading. 


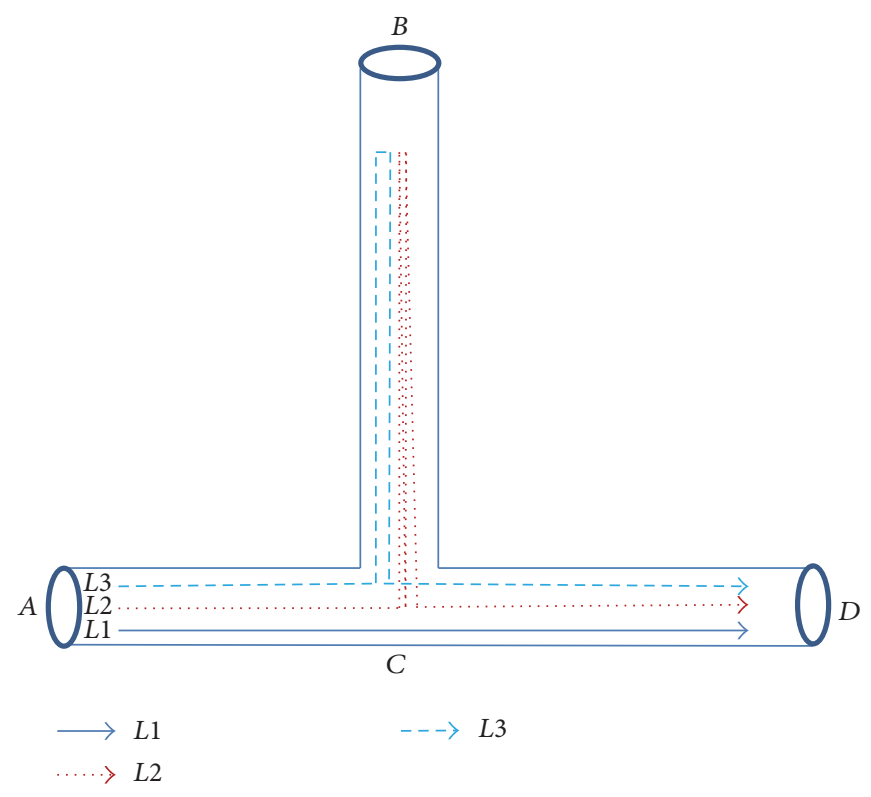

FIgURE 3: Branch circuit of power line communication.

Not only is the signal on power line directly transmitted from the sending node $A$ to the receiving node $D$, but also it reflected many times to arrive at the receiving node $D$, forming the multipath effect. The signals in Figure 3 may have the transmission path as follows: (1) $L 1: A \rightarrow C \rightarrow D$; (2) $L 2: A \rightarrow C \rightarrow B \rightarrow C \rightarrow B \rightarrow C \rightarrow D$; (3) L3: $A \rightarrow C \rightarrow B \rightarrow C \rightarrow D$.

Since the multipath effect disperses the signal ability and arrives at the receiver with the different signal phase, it seriously influences the accepted effect. When there are $n$ paths, frequency diversity can be achieved by changing the carrier frequency to improve the signal decision rate.

We suppose the channel transfer function of the $i$ th path in multipaths is $H_{i}(f)$, so transfer function of the power line channel was formulated as follows:

$$
H(f)=\sum_{i} H_{i}(f)
$$

where $H(f)$ present the superposition of multipaths transmission characteristics. According to the reasons of multipath transmission, we analyzed the transmission characteristics on each path. There has been a path delay and the signal is attenuated with the increase of the transmission distance and frequency [18].

According to formula (1), we consider the main parameters affecting the characteristics of each path to establish the power line channel model. Meanwhile, for simulation, we simplify (1) as follows [19]:

$$
H(f)=\sum_{i=1}^{N} g_{i}(f) e^{-\left(\alpha_{0}+\alpha_{1} f^{k}\right) d_{i}} e^{-j 2 \pi f \tau_{i}},
$$

in which parameters $\left(a_{0}, a_{1}\right.$, and $\left.k\right)$ are obtained by field measurements on the reference channel. Coefficient $g_{i}(f)$ is the weight of each multipath channel generated randomly

\begin{tabular}{|c|c|}
\hline Model parameters & References \\
\hline$i$ & $\begin{array}{l}\text { The number of paths; when the path is the } \\
\text { shortest, } i=1\end{array}$ \\
\hline$a_{1}, a_{2}$ & Attenuation parameter \\
\hline$k$ & $\begin{array}{l}\text { Attenuation factor index; the typical value is } \\
\qquad 0.5 \sim 1\end{array}$ \\
\hline$g_{i}$ & $\begin{array}{l}\text { Weighted factor of path } i \text {; its absolute value } \\
\text { is less than or equal to } 1\end{array}$ \\
\hline$d_{i}$ & The length of path $i$ \\
\hline$\tau_{i}$ & The delay of path $i$ \\
\hline$f$ & Frequency \\
\hline
\end{tabular}

TABLE 1: Parameters of transfer function model.

between $(-1,1)$. The parameter $d_{i}$ is the channel length of each path, with uniform distribution on $(0, L)$. $L$ defined the upper limit of the path length on each scenario. In the power line channel model, main parameters in Table 1 can be used for modeling and analyzing the typical power line channel $[8,20]$. Although we reduce parameters, they also can reflect the power line channel characteristics.

The mathematical model of power line channel shows that the power line channel is a multipath channel, which has frequency selective fading. The frequency response function reveals the sparse nature of the transmission characteristics of the power line channel.

\section{Compressive Sensing Estimation Channel Characteristics}

Due to the electromagnetic phenomena and other external causes, the environments usually affect the performance of 


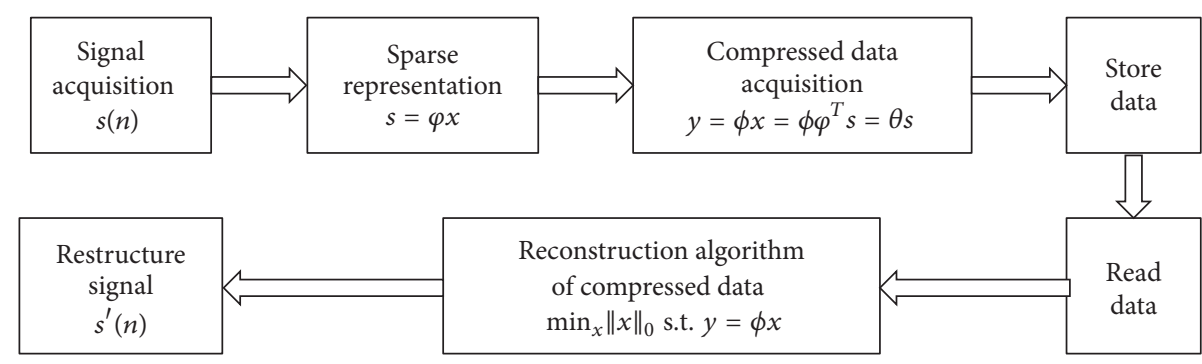

Figure 4: Process of CS.

device in power line communication. Therefore, it is very necessary to evaluate the channel accurately to acquire characteristic parameters of the channel impulse response.

3.1. Compressive Sensing Technology. The process of compress sensing includes three steps: signal sparse representation, data compression, and reconstruction of compressed data as shown in Figure 4.

The compression sensing is a novel information acquisition theory, which mainly consists of two parts: the signal is projected on the measurement vector to obtain the measured value; the signal is reconstructed from the measured value. Usually, the power steady-state harmonic signal can be expressed as

$$
f(t)=a_{0} \cos \left(2 \pi \gamma_{0} t+\theta_{0}\right)+\sum_{m=1}^{L} a_{m} \cos \left(2 \pi \gamma_{m} t+\theta_{m}\right),
$$

where $a_{0}, \gamma_{0}$, and $\theta_{0}$ are the amplitude, frequency, and phase of the fundamental component; $L$ denotes the number of harmonics; $a_{m}, \gamma_{m}$, and $\theta_{m}$ denote the amplitude, frequency, and phase of the $m$ th harmonic component. We assume the signal $f(t)$ to be $s$ in time domain. And then, we can start the process of compress sensing as follows.

3.1.1. Signal Sparse Representation. Assume the signal $s$ has no sparsity in its time domain; it can be converted to another domain to obtain the sparsity projection $x$. Then, we can compress the sparsity $x$ by the compress sensing technology. The transform domain projection process is the process of signal sparse representation, as shown in

$$
s=\varphi x,
$$

where $s$ is the original signal without sparsity; $\varphi$ denotes the projection matrix; $x$ is the projection of $s$ in the projection matrix $\varphi$, that is, the sparse signals.

3.1.2. Data Compression. Let $x(n)$ be a digital signal sampled by the ADC; the dimension is $N$. If $x$ is a sparse signal and the sparsity is $K(K \ll N)$, that is, there are only $K$ nonzero elements in the signal, then it can be compressed by the compressed sensing technology to reduce the dimension $N$ of the original signal $x(n)$ to the dimension $M(M \ll N)$ and then we can get the compressed signal $y$ as shown in

$$
y=\phi x=\phi \varphi^{T} s=\theta s,
$$

where $y$ is the compressed signal; $\phi$ is an opposite observation matrix; $x$ is the discrete signal; $s$ is the original signal.

3.1.3. Reconstruction of Compressed Data. Since the front-end hardware completed the data compression process, it reduces the storage requirements of analysis section. Then, we just focus on the back-end hardware compression algorithm for data recovery reconstruction work. Based on the above compression algorithm, the restore reconstructed of the compressed signal can be realized through 0 -minimum-norm by

$$
\begin{array}{ll}
\min _{x} & \|x\|_{0} \\
\text { s.t. } & y=\phi x,
\end{array}
$$

where $x$ denotes the sparse signal with reconstruction; $y$ is the restored signal after $x$ 's observations; 0 -minimum-norm indicates the number of nonzero elements. The results of CS in sampling are as shown in Figure 5.

3.2. CS Channel Estimation. The traditional least squares channel estimation response is equal length pilot blocks by transmitting the channel impact, so that the transmitted signal and channel impulse response of linear convolution are converted to circular convolution; received vector can be written as

$$
y=p * h+n
$$

where $*$ denotes the circular convolution; $P$ is the transmitted pilot signal.

$$
y=C h+n
$$

After constructing an appropriate pilot signal, it transmits the test pilot signal $C$ and couples power line at the transmitting terminal through couplers. However, the pilot signal is affected due to channel transmission characteristics of the power line and the power line noise. Figure 6 shows the estimation model in the pilot point. 


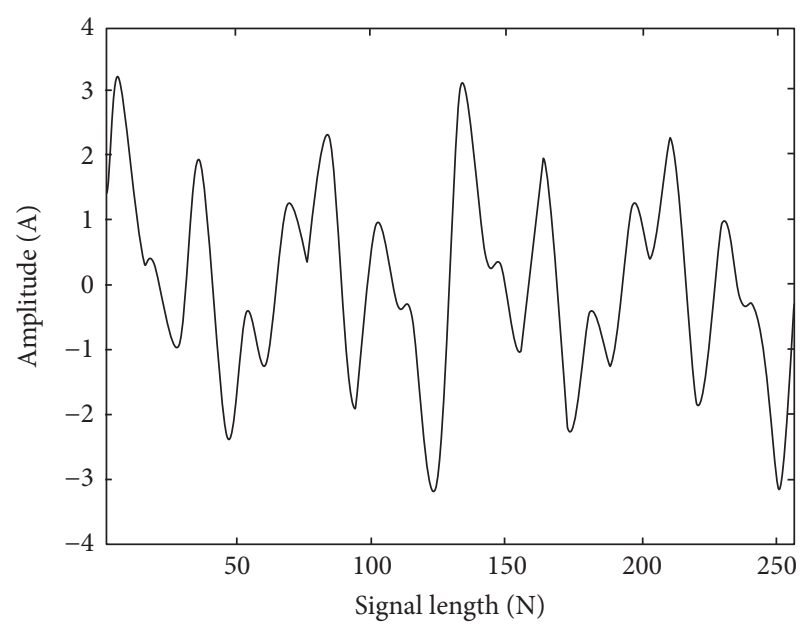

(a) The original signal

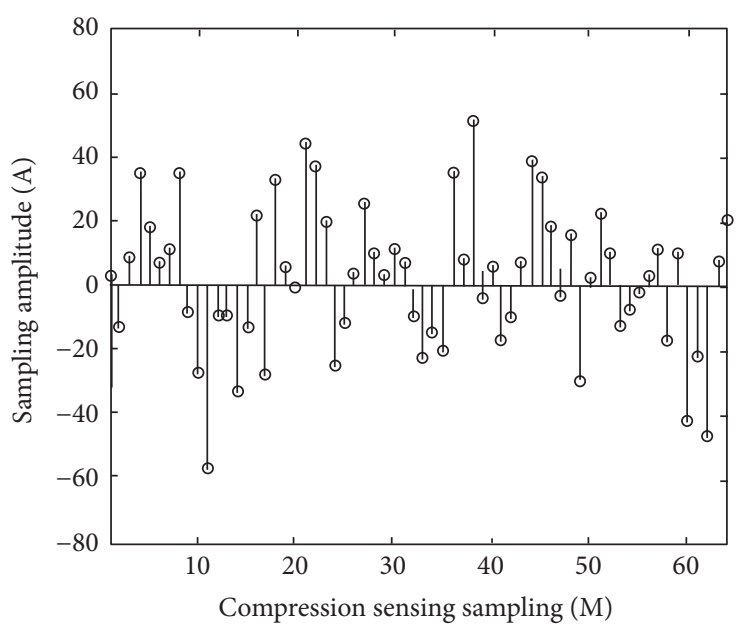

(b) The sampling signal by CS

FIGURE 5: Sampling process of CS.

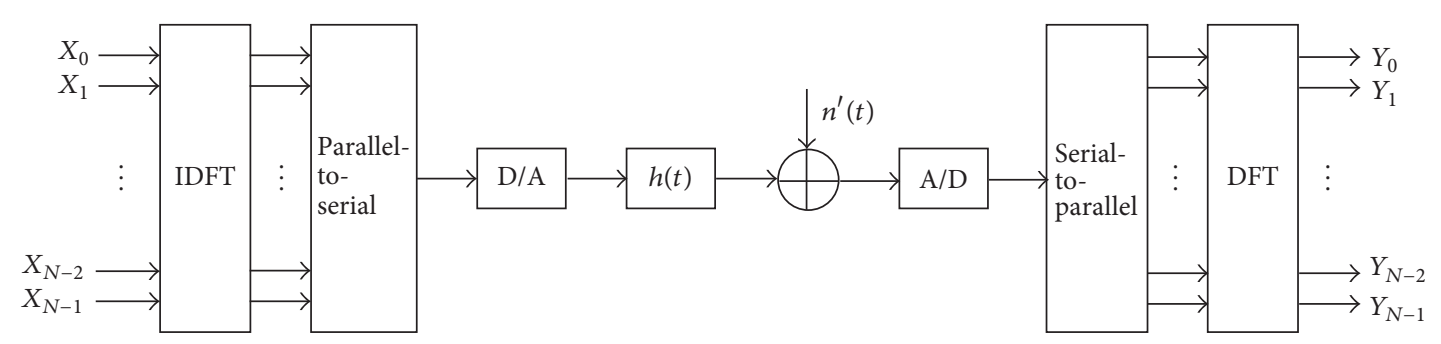

FIGURE 6: Pilot point channel estimation model.

Therefore, we just focus on the model and disturb signal by noise in the receiving end through couplers as described in

$$
\begin{aligned}
{\left[\begin{array}{c}
y_{0} \\
y_{1} \\
\vdots \\
y_{M-2} \\
y_{M-1}
\end{array}\right]_{M \times 1}=\left[\begin{array}{ccc}
c_{0} & & c_{1} \\
c_{1} & \ddots & c_{2} \\
\vdots & & \vdots \\
c_{M-1} & \ddots & c_{M} \\
c_{M} & & c_{0}
\end{array}\right]_{M \times N}\left[\begin{array}{c}
h_{0} \\
h_{1} \\
\vdots \\
h_{N-2} \\
h_{N-1}
\end{array}\right]_{N \times 1} } \\
+\left[\begin{array}{c}
n_{0} \\
n_{1} \\
\vdots \\
n_{M-2} \\
n_{M-1}
\end{array}\right]_{M \times 1}
\end{aligned}
$$

Assume the available power line communication channel characteristics $y$ are at receiving end; we employ the sensing reconstruction algorithm (OMP, Orthogonal Matching Pursuit Algorithm) to estimate the power line channel impulse response $h(t)$.

OMP algorithm is the improved algorithm of MP algorithm. OMP algorithm selects and observes the most closely matched atom signal from the atom library as OM algorithm. The orthogonality will make the selected atom not be repeated in the OMP algorithm iterative process, which ensures the optimality of iteration and thereby reduces the number of iterations and good reconstruction.

The restored reconstruction of OMP is as follows:

(1) Initialization is as follows: residual value $r_{0}=y$, index set $\Lambda_{0}=\Phi$, and iterations $i=1, \Gamma_{0}=\Phi$.

(2) Determine index value: $\lambda_{i+1}=\arg \max \left|\left\langle r_{i}, \tau_{j}\right\rangle\right|$, where $\tau_{j}$ is column $j$ on matrix $\Phi$; determine the position of the corresponding atom, that is, the position of nonzero element: $\left\{\Lambda_{i+1}=\Lambda_{i} \cup \lambda_{i+1}\right\},\left\{\Gamma_{i+1}=\right.$ $\left.\Gamma_{i} \cup \tau \lambda_{i+1}\right\}$.

(3) LS algorithm is used to obtain new estimates:

$$
\widehat{x}_{i+1}=\arg \min \left\|y-\Gamma_{i+1} \widehat{x}\right\|=\Gamma_{i+1}{ }^{+} x .
$$

in which + is devoted pseudoinverse.

(4) Calculate the new residual value: $r_{i+1}=y-\Gamma_{i+1} \widehat{x}_{i+1}$.

(5) Optimize the iterative process: construct loop $i=$ $i+1$, and then repeat the indexing process until the completion of the required number $m$ of iterations to terminate the iteration.

(6) Complete signal reconstruction: the calculated estimated value satisfies equality as $\widehat{x}_{\Lambda_{m}}=\widehat{x}_{m}$ and $\widehat{x}_{\{1, \ldots, 2 N\}-\Lambda_{m}}=0$. 


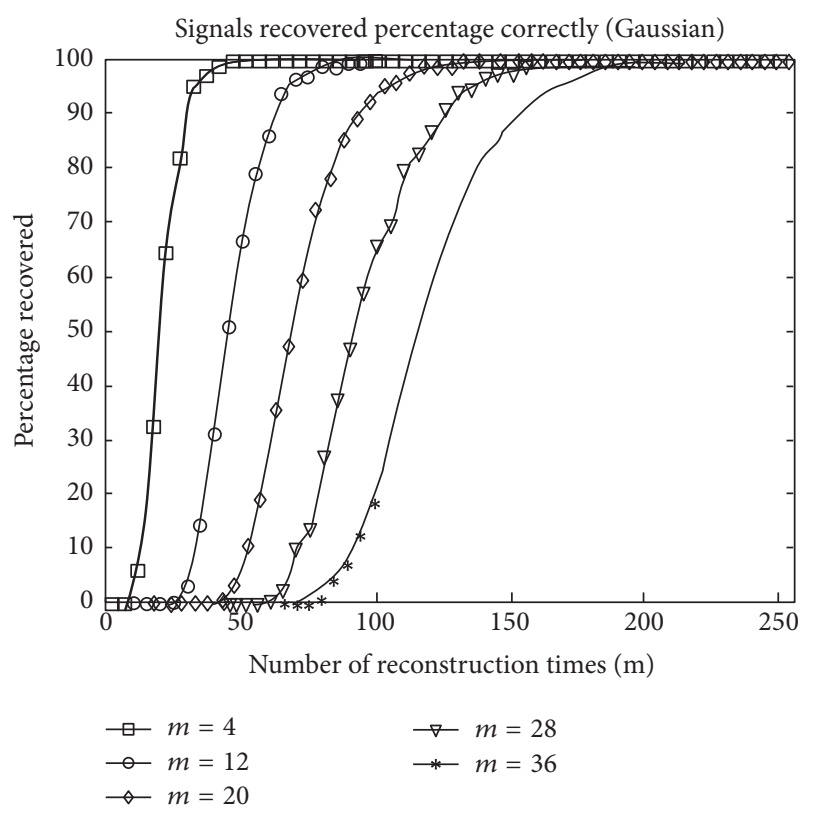

Figure 7: OMP multireconstruction.

TABLE 2: Parameters list.

\begin{tabular}{lc}
\hline Parameters & Value \\
\hline Bandwidth simulation & $B_{W}=30 \mathrm{MHz}$ \\
Sampling frequency & $f_{s}=60 \mathrm{MHz}$ \\
Sampling time & $t=10 \mu \mathrm{s}$ \\
\hline
\end{tabular}

In each interaction, OMP algorithm obtains a nonzero element corresponding position in $x$ and calculates the value of this element. After $m$ iterations, it can get the estimation value $\widehat{x}$, and then it estimates the power line channel impulse response. The reconstruction is as shown in Figure 7.

\section{Simulation Result}

Based on Matlab simulation platform, we built the simulation environment and simulated the power line channel impulse response. PLC channel frequency domain response is in 15 paths, and the longest path is $1000 \mathrm{~m}$ in the channel model. And based on the least squares channel estimation of the pilot sequences and CS-based power line channel estimation, we analyze the simulation results under the same conditions.

To build the simulation environment, we refer to the reference communication channel parameters and simulate the time-domain characteristics of power line reference communication channel. The parameters are shown in Table 2.

4.1. Different SNR (Signal to Noise Ratio). Power line noises are very complex; they can be roughly divided into five categories in the time domain: the colored background noise, narrowband noise, asynchronous to power frequency periodic impulse noise, synchronized to power frequency periodic impulse noise, and sudden impulse noise.

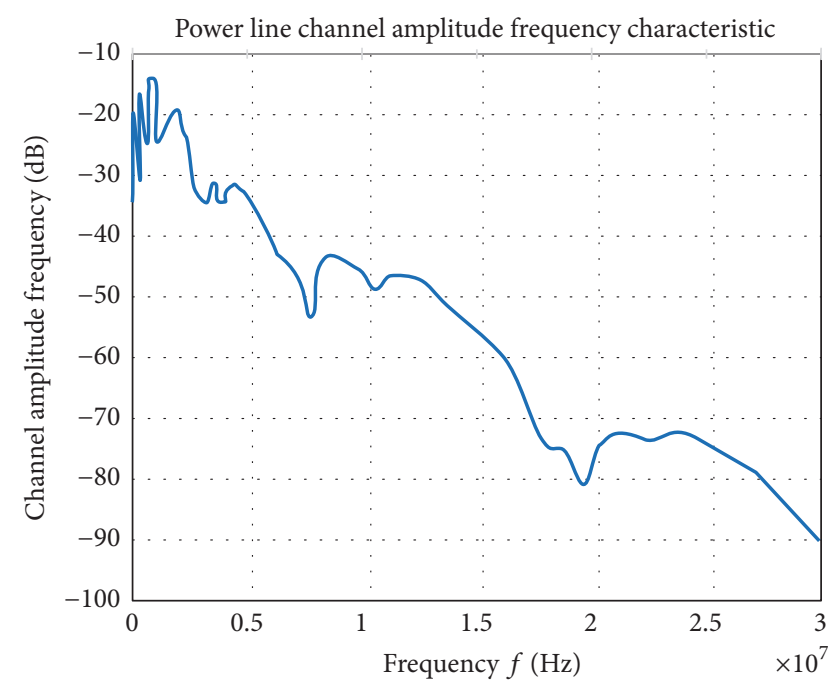

FIGURE 8: Amplitude frequency response of reference channel.

In implementation of the project, the average power of noises, the colored background noise, narrowband noise, and asynchronous to power frequency periodic impulse noise, are small. And these three noises time-varying characteristics are weak; they change slowly with time in the entire PLC carrier communication frequency band. Thus, they can be called background noise. Meanwhile, the average power synchronization frequency periodic pulse noise and sudden impulse noise are relatively large. And there are two noises changes, frequently and randomly. They thus are called random impulse noise. Although the random impulse noise appears little, this type of noise greatly affects the quality of communication. It usually causes the narrowband communication interruption. Therefore, we just consider the superposition persistence of background noise in the channel in simulation.

Based on the impulse response of power line channel, we built the suitable pilot signal matrix. The dimensions of fixed reception signal are as follows: $M 50$ and $N 200$. And due to the interference of power line channel by background noise, the signal to noise ratio changes are from $5 \mathrm{~dB}$ to $30 \mathrm{~dB}$.

As shown in Figures 8 and 9, compared to the traditional least squares channel estimation, CS channel estimation has a better estimation by the compressed sensing technology in the low SNR and hostile environment. The estimation error is less than tenfold the traditional least squares channel estimation algorithm. As the SNR increases and the channel environment tends to be better, the least squares channel estimation and compressive sensing-based channel estimation can achieve good estimation. However, compared to compressive sensing-based channel estimation, the least squares estimation needs longer pilot signal, more data computation, and longer calculation time.

4.2. Different Compression Dimension. Based on the impulse response of power line channel, we built the suitable pilot signal matrix again. For the dimensions of fixed reception signal, we increase $M$ from 50 to 150 gradually and $N$ is at 200. 


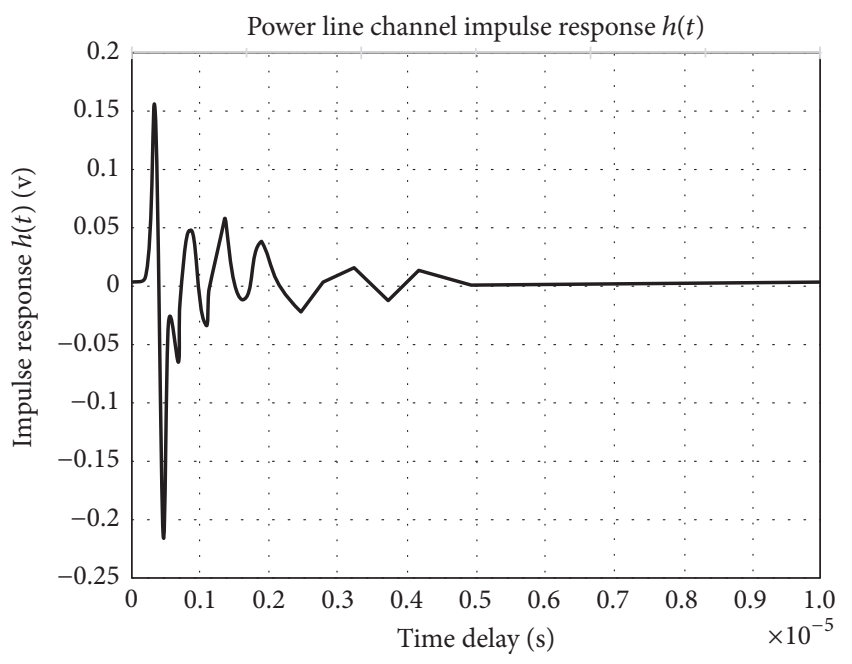

FIGURE 9: Impulse response of reference channel.

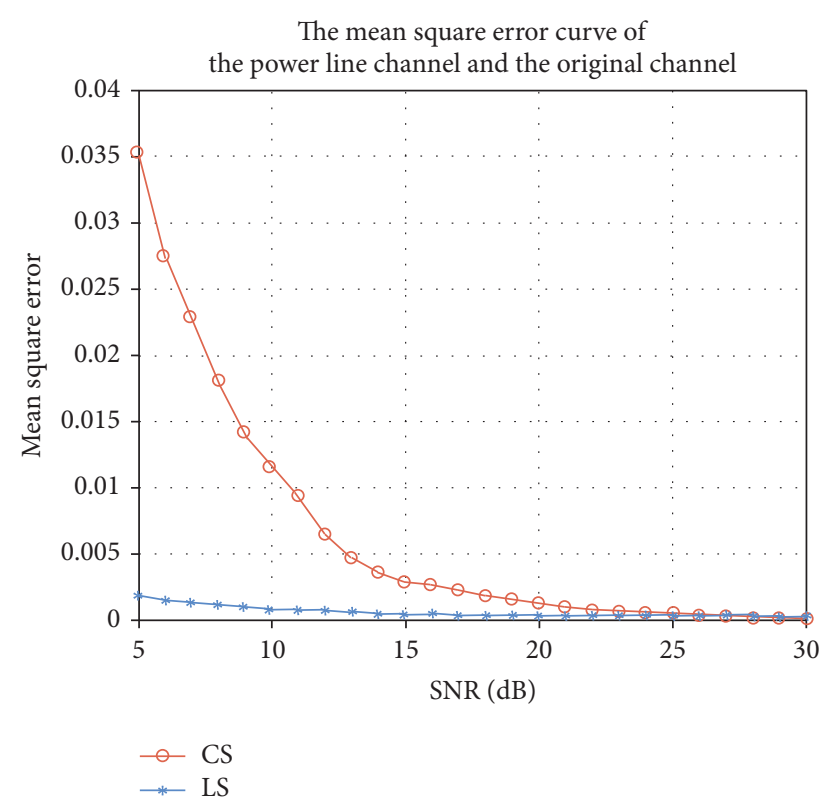

FIgURE 10: Performance comparison of CS and LS (SNR).

Meanwhile, we added the noise power line channel and fixed the noise ratio at $15 \mathrm{~dB}$.

As shown in Figures 10 and 11, in the unchanged situation of the power line signal to noise ratio, with the increasing of received signals' dimension of $M$, these two methods performance of power line channel estimation is improved. However, due to the sparsity of power line channel, CS-based power line channel estimation adopts the sparsity to improve the efficient and accurate estimation of the power line channel characteristics, which achieves better channel restoration reconstruction.

However, with the growing pilot sequences, the pilot signal-based least squares channel estimation method can achieve good channel estimation. However, compared to the former, the compressed sensing-based power line channel

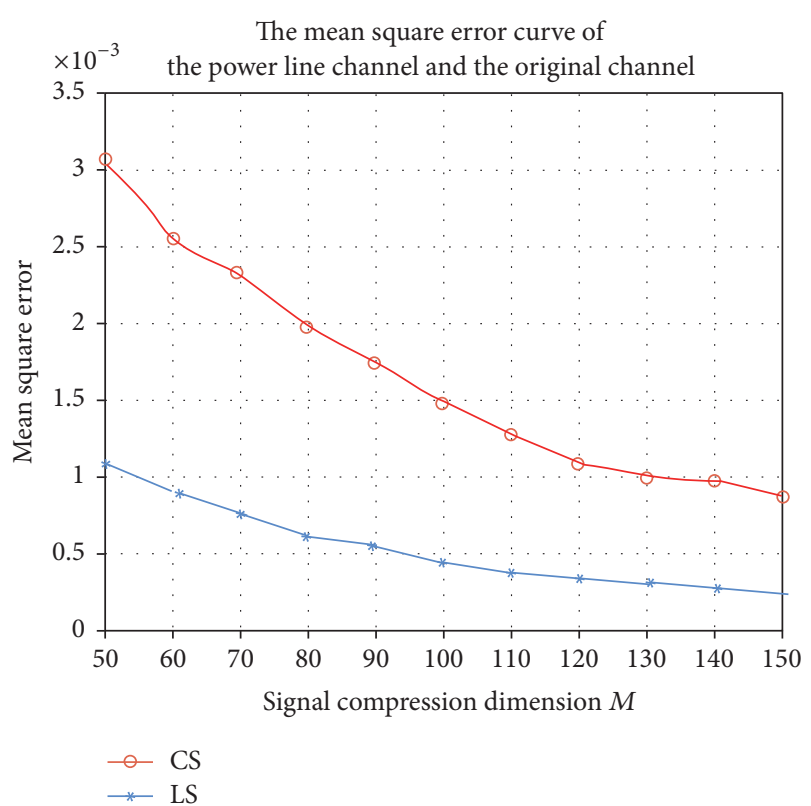

FIGURE 11: Performance comparison of CS and LS (M).

estimation algorithm has advantages and achieves compression objective data.

\section{Conclusion}

In this paper, we analyzed the sparsity of power line channel firstly. On this basis, we proposed a method of power line channel estimation based on compressive sensing. The simulation results show that this method used less pilot signal and fewer storage resources. However, the performance of the algorithm is better than the least square channel estimation algorithm. Therefore, the algorithm proposed in this paper has better application prospect.

In the future, we will still research various algorithms to improve the efficiency in compression sensing.

\section{Conflicts of Interest}

The authors declare that there are no conflicts of interest regarding the publication of this paper.

\section{References}

[1] M. Yigit, V. C. Gungor, G. Tuna, M. Rangoussi, and E. Fadel, "Power line communication technologies for smart grid applications: a review of advances and challenges," Computer Networks the International Journal of Computer \& Telecommunications Networking, vol. 70, no. 10, pp. 366-383, 2014.

[2] S. K. Shen, "Power line communication system and control method thereof," 2016.

[3] A. J. Hicks Iii, B. Davis, G. Howell et al., "Power-line communications," US20160148499, 2016.

[4] B. M. Propp and D. L. Propp, "Power line communication apparatus," U.S. Patent 4,815,106. 1989. 
[5] M. Götz, M. Rapp, and K. Dostert, "Power line channel characteristics and their effect on communication system design," IEEE Communications Magazine, vol. 42, no. 4, pp. 78-86, 2004.

[6] M. Zamani, Z. Zhang, and C. Li, "Channel estimation for optical orthogonal frequency division multiplexing systems," 2016.

[7] P. L. Zhang, H. X. Zhang, H. D. Liu, Y. J. Zhang, P. F. He, and X. L. Pang, "Particle filtering based channel estimation in OFDM power line communication," Journal of China Universities of Posts \& Telecommunications, vol. 21, no. 5, pp. 24-30, 2014.

[8] B. Yunus and H. Li, "Analysis of power quality waveform for data transmission efficiency over IEC 61850 communication standard," in Proceedings of the 1st International Power and Energy Conference (PECon '06), pp. 161-166, November 2006.

[9] C. Tse, "Power quality meter and method of waveform anaylsis and compression," 2015.

[10] A. Rahim Abdullah, H. T. N. Ahmad, A. N. Abidullah et al., "Performance evaluation of real power quality disturbances analysis using s-transform," Applied Mechanics \& Materials, vol. 753, no. 2015, pp. 1343-1348, 2015.

[11] D. L. Donoho, "Compressed sensing," IEEE. Transactions on Information Theory, vol. 52, no. 4, pp. 1289-1306, 2006.

[12] R. G. Baraniuk, "Compressive sensing," IEEE Signal Processing Magazine, vol. 24, no. 4, pp. 118-124, 2007.

[13] J. A. Tropp and A. C. Gilbert, "Signal recovery from random measurements via orthogonal matching pursuit," IEEE. Transactions on Information Theory, vol. 53, no. 12, pp. 4655-4666, 2007.

[14] D. Tralic and S. Grgic, "Signal reconstruction via compressive sensing," in Proceedings of the 53rd International Symposium (ELMAR '11), pp. 5-9, Zadar, Croatia, September 2011.

[15] S. C. Yan and J. Qi, "Research on service impact analysis for power communication network based on N-1 principle," Advanced Materials Research, vol. 846-847, pp. 396-399, 2014.

[16] J. Y. Shin and J. C. Jeong, "Power line channel model considering adjacent nodes with reduced calculation complexity due to multipath signal propagation and network size using infinite geometric series and matrices," Transactions of the Korean Institute of Electrical Engineers, vol. 58, no. 2, pp. 248-255, 2009.

[17] A. Tomasoni, R. Riva, and S. Bellini, "Spatial correlation analysis and model for in-home MIMO power line channels," in Proceedings of the 16th IEEE International Symposium on Power Line Communications and Its Applications (ISPLC '12), pp. 286291, March 2012.

[18] J. Matanza, S. Alexandres, and C. Rodríguez-Morcillo, "Advanced metering infrastructure performance using European low-voltage power line communication networks," IET Communications, vol. 8, no. 7, pp. 1041-1047, 2014.

[19] Y. Xiao, J. Zhang, F. Pan, and Y. Shen, "Power line communication simulation considering cyclostationary noise for metering systems," Journal of Circuits, Systems and Computers, vol. 25, no. 9, Article ID 1650105, 2016.

[20] Y. Wang, Q. D. Wang, X. Z. Hou, H. L. Sun, X. M. Chen, and X. J. Li, "Measurement and research on attenuation characteristics of low voltage power line communication channel," Advanced Materials Research, vol. 986-987, pp. 2068-2072, 2014. 


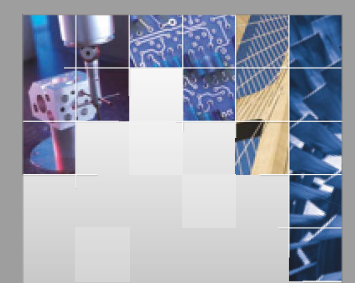

\section{Enfincering}
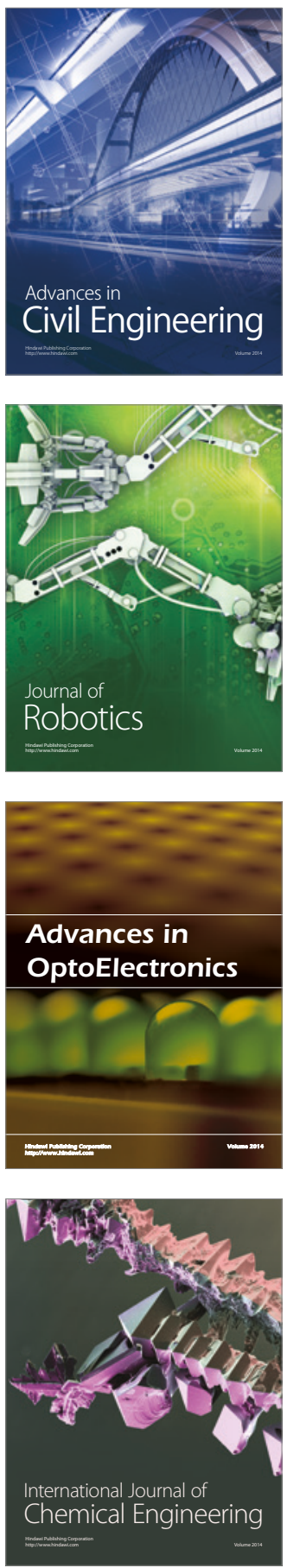

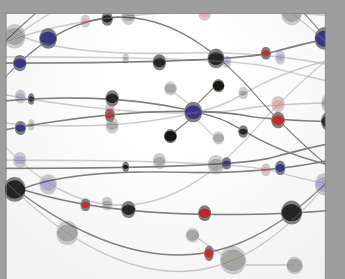

The Scientific World Journal

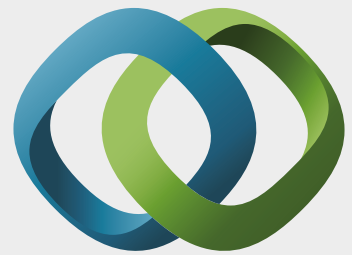

\section{Hindawi}

Submit your manuscripts at

https://www.hindawi.com
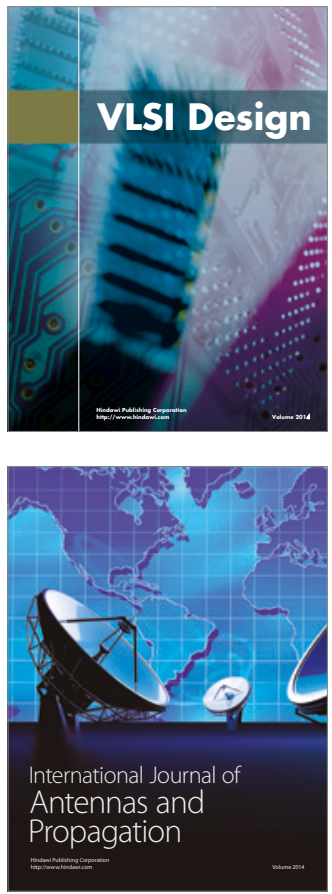

\section{Rotating}

Machinery
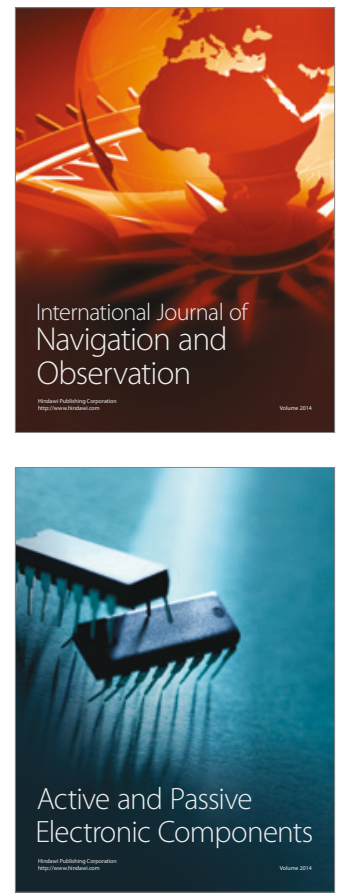
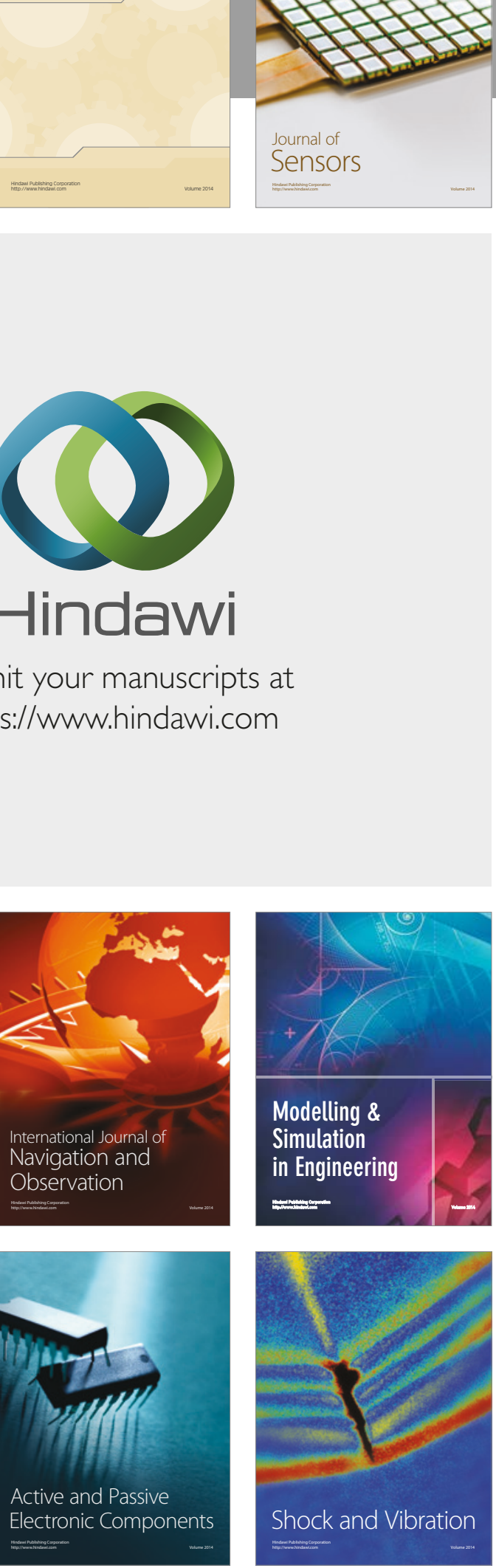
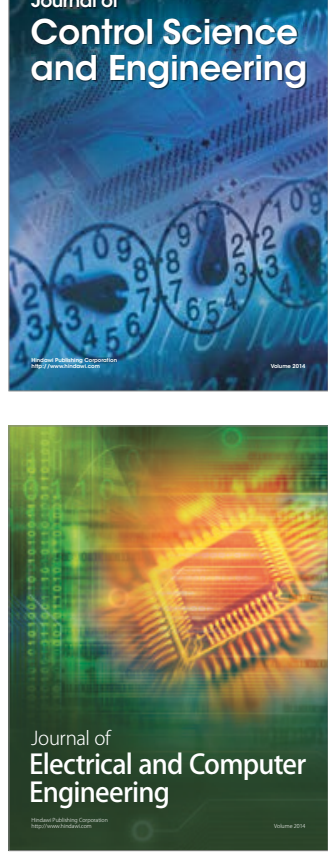

Distributed

Journal of

Control Science

and Engineering
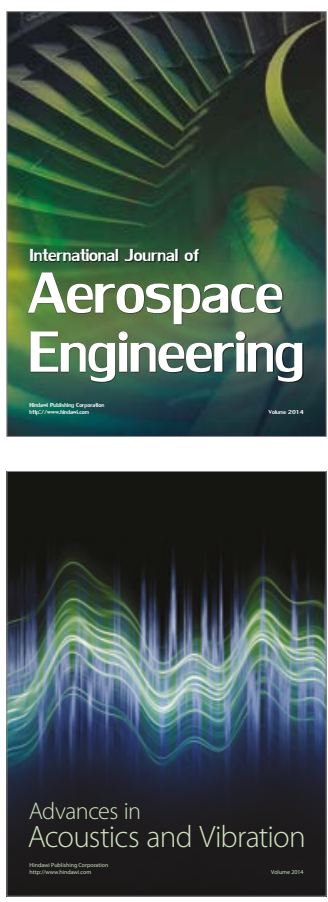

Sensor Networks 